\section{Representation and Recognition of Handwritten Digits Using Deformable Templates}

\author{
Anil K. Jain, Fellow, IEEE, and Douglas Zongker
}

\begin{abstract}
We investigate the application of deformable templates to recognition of handprinted digits. Two characters are matched by deforming the contour of one to fit the edge strengths of the other, and a dissimilarity measure is derived from the amount of deformation needed, the goodness of fit of the edges, and the interior overlap between the deformed shapes. Classification using the minimum dissimilarity results in recognition rates up to 99.25 percent on a 2,000 character subset of NIST Special Database 1. Additional experiments on an independent test data were done to demonstrate the robustness of this method. Multidimensional scaling is also applied to the 2,000 $\times$ 2,000 proximity matrix, using the dissimilarity measure as a distance, to embed the patterns as points in low-dimensional spaces. A nearest neighbor classifier is applied to the resulting pattern matrices. The classification accuracies obtained in the derived feature space demonstrate that there does exist a good low-dimensional representation space. Methods to reduce the computational requirements, the primary limiting factor of this method, are discussed.
\end{abstract}

Index Terms-Digit recognition, deformable template, feature extraction, multidimensional scaling, clustering, nearest neighbor classification.

\section{INTRODUCTION}

AUTOMATIC recognition of handprinted characters has long been a goal of many research efforts in the pattern recognition field [4]. The subproblem of digit recognition is also seen as important, not only because advances in it are expected to lead to advances in the general case, but also because of its immediate applicability to a number of fields, the most frequently cited of which is the reading of postal ZIP codes from mail pieces.

The challenges in handwritten digit recognition arise not only from the different ways in which a single digit can be written, but also from the varying requirements imposed by the specific applications. The primary performance measures are classification accuracy and recognition speed-a system for reading ZIP codes from envelopes may not be appropriate for reading amounts from checks, due to the differing volumes and costs of error.

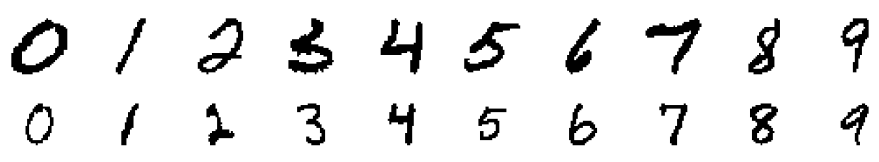

Fig. 1. Sample digit images: top row, from the NIST FL-3 data set; bottom row, from the IBM data set. Character size is $32 \times 32$ for NIST data and $16 \times 24$ for IBM data.

A number of schemes for digit classification have been reported in the literature. They differ in the feature extraction and classifica-

- A.K. Jain is with the Department of Computer Science, Michigan State University, East Lansing, MI 48824. E-mail: jain@cps.msu.edu.

- D. Zongker is with the Department of Computer Science and Engineering, University of Washington, Seattle, WA 98195-2350.

E-mail: dougz@cs.washington.edu.

Manuscript received 16 Aug. 1996; revised 1 Aug. 1997. Recommended for acceptance by M. Mohiuddin.

For information on obtaining reprints of this article, please send e-mail to: tpami@computer.org, and reference IEEECS Log Number 105475. tion stages employed. Many methods for extracting features from character images have been proposed. The proposed features include counts of topological features (crossings, endpoints, holes, etc.) and various mathematical moments. While these ad hoc features have performed well in many tests, they are neither intuitive nor, in many cases, generally applicable to other character sets. Classification methods used for digit recognition include nearest neighbor classifiers and multilayer perceptron networks. There has also been a recent trend to combine the outputs of multiple classifiers [13].

A more intuitive alternative to these feature extraction models is the use of deformable templates, where an image deformation is used to match an unknown image against a database of known images. We have investigated the use of image deformation to handprinted digit recognition. Therefore, our literature review includes only similar approaches; for a wider survey of digit recognition in general, refer to the recent paper by Trier et al. [14].

The goal of this paper is to investigate the deformation of character image outlines as a source of information for recognition. We show that a combination of the deformation energy required to match two character images and the template matching coefficients of the resulting binary images form a good measure of dissimilarity between images. After reviewing other techniques for deformation-based matching, we present our deformation model, discuss the use of this model for feature extraction, and present results with this method on a 2,000 image NIST SD-1 handwritten digit data set. (The NIST images we have worked with are from the FL-3 distribution, a subset of SD-1 containing approximately 3,500 digit images.) We have also investigated using the NIST data as a training set for classifying 2,000 digit images from another database, provided by IBM Almaden Research Center.

\section{Deformable Models for Digit Recognition}

A number of studies have been reported in the literature which have applied deformable models to digit recognition. Research in this area has concentrated on taking a skeletonized digit image, representing it with a number of curve segments, and then altering the curve parameters to deform the image. Nishida [9] proposes a grammar-like model for applying deformations to structures composed of primitive strokes. Lam and Suen [8] use a two-stage method for recognition, in which samples are first classified by their structure using a tree classifier. Samples which can not be satisfactorily assigned to a class in this way are passed to a slower relaxation matching algorithm which uses deformation to match the sample to each template. They report a 93.15 percent recognition rate, with a 4.60 percent rejection rate on a 2,000-sample database taken from USPS ZIP code images. Cheung et al. [2] model characters with a spline, and assume that the spline parameters have a multivariate Gaussian distribution. A Bayesian approach is then used to determine the character class, with the model parameters as prior and the image data parameters as likelihood. This method achieved a 95.4 percent recognition rate on the NIST SD-1 handprinted digit set. Revow et al. [10] model digits as inkgenerating Gaussian "beads" strung along a spline outline. Characters are matched through deformation of the spline and adjustment of the bead parameters. Their best result reported has 99.00 percent recognition accuracy on a 2,000 character set with no rejections.

Simard et al. [11] present a digit recognition system based on an efficient distance measure that is locally invariant to transformations such as translation, rotation, scaling, stroke thickness, and others. Efficiency is further improved by using a multiresolution algorithm to differentiate very dissimilar patterns using a simpler, coarser distance measures. On a NIST-provided set of 60,000 training patterns and 10,000 test patterns, this method reached a 0.7 percent error rate. 


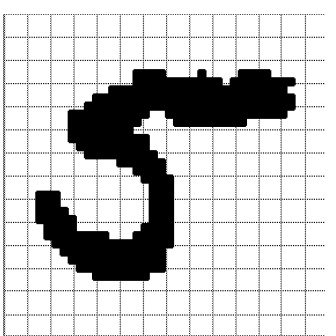

(a)

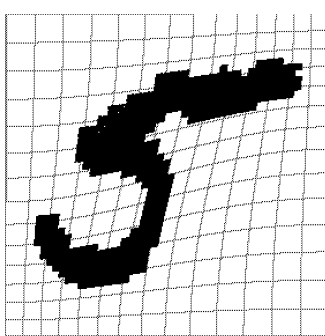

(b)

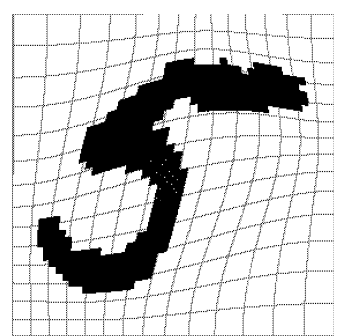

(c)

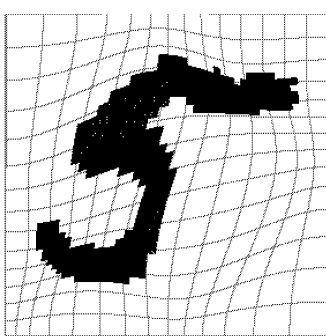

(d)

Fig. 2. Deformations of a sample digit image. (a) Original image. (b) $M=N=1$. (c) $M=N=2$. (d) $M=N=3$.

Casey [1] gives a method for linear transformation of digit images, based on moment normalization, for removing some skew and orientation variation. This is used as a preprocessing step by Gader et al. [3] for a digit recognition system based on binary template matching. The authors report recognition rates in the range of 94.03-96.39 percent, with error rates in the range 0.54-1.05 percent.

Wakahara [16] uses iterated local affine transformation (LAT) operations to deform binary images to match prototype digit images. This method correctly identified 96.8 percent of the characters in a 2,400-sample database, with a substitution error rate of 0.2 percent and a reject rate of 3 percent.

The deformation and matching technique used in this paper was proposed by Jain et al. [6]. In this approach, the image is considered to be mapped to the unit square $S=[0,1]^{2}$. The deformation is then represented by a displacement function $D(x, y)$. These displacement functions are continuous and are zero on the edges of the unit square. The mapping $(x, y) \mapsto(x, y)+D(x, y)$ is thus a deformation of $S$, a smooth mapping of the unit square onto itself. The space of displacement functions has an infinite orthogonal basis:

$$
\begin{aligned}
& \mathbf{e}_{m n}^{x}(x, y)=(2 \sin (\pi n x) \cos (\pi m y), 0) \\
& \mathbf{e}_{m n}^{y}(x, y)=(0,2 \cos (\pi m x) \sin (\pi n y))
\end{aligned}
$$

for $m, n=1,2, \ldots$ Low values of $m$ and/or $n$ correspond to lower frequency components of the deformation in the $x$ and $y$ directions, respectively. Fig. 2 shows a series of deformations using progressively higher-order terms. Note that the deformation gets more severe as higher-order terms are included in the expansion. A parameter vector $\xi$ can be used to represent a specific deformation function with the following basis:

$$
D_{\xi}(x, y)=\sum_{m=1}^{\infty} \sum_{n=1}^{\infty} \frac{\xi_{m n}^{x} \mathbf{e}_{m n}^{x}+\xi_{m n}^{y} \mathbf{e}_{m n}^{y}}{\lambda_{m n}} .
$$

The parameters $\lambda_{m n}=\alpha \pi^{2}\left(n^{2}+m^{2}\right)$ serve as normalizing constants.

\section{Methodology}

The basic goal is to determine the dissimilarity between two digit images using a deformable template approach. This is achieved by transforming one image into a template, and deforming it to fit the other image as closely as possible. The dissimilarity measure is defined in terms of

1) how well the deformed template fits the target image and

2) how much deformation was required.

In practice, we truncate the infinite series expansion of (3) to get a finite-length parameter vector $\xi$ :

$$
D_{\xi}(x, y)=\sum_{m=1}^{M} \sum_{n=1}^{N} \frac{\xi_{m n}^{x} \mathbf{e}_{m n}^{x}+\xi_{m n}^{y} \mathbf{e}_{m n}^{y}}{\lambda_{m n}} .
$$

The template is fit to the target image using a Bayesian approach, as in [6]. The prior is a function of $\xi$, a measure of how much deformation of the template is required.
We use $M$ and $N$ equal to 3 . This choice allows a sufficiently wide range of possible deformations, while keeping the number of parameters, and hence the computational requirements, reasonable. The parameter vector $\xi$ consists of nine ordered pairs. A probability density is assumed for the components of $\xi$. For simplicity, we assume that the terms $\xi_{m n}$ are independent of each other, that the $x$ and $y$ components are independent, and that they are each Gaussian distributed with mean zero and variance $\sigma^{2}$. This leads to the following prior distribution on the parameter vector:

$$
P(\xi)=\kappa \frac{1}{\left(2 \pi \sigma^{2}\right)^{M N}} \exp \left\{-\frac{1}{2 \sigma^{2}}\left[\sum_{m=1}^{M} \sum_{n=1}^{N}\left(\left(\xi_{m n}^{x}\right)^{2}+\left(\xi_{m n}^{y}\right)^{2}\right)\right]\right\},
$$

where $\kappa$ is a normalizing constant.

The likelihood is determined by how well the template contour fits the edge location and direction of the target image (as determined by the Canny edge detection operator). This is given by an energy function defined at the points of the deformed template $T \xi$, in terms of the deformation vector and the target image $Y$ :

$$
E\left(T_{\xi}, Y\right)=\frac{1}{n_{T}} \sum(1+\Phi(x, y)|\cos (\beta(x, y))|) \text {. }
$$

Here, $n_{T}$ is the number of pixels in the template outline, $\Phi(x, y)$ is an edge potential function (lowest near edge pixels in the target image $Y)$, and $\beta(x, y)$ is the angle between the tangent direction of the template at $(x, y)$ and the tangent direction of the nearest edge in the target direction.

We combine the prior probability and likelihood using Bayes rule to derive the following objective function, which we attempt to minimize:

$$
O\left(T_{\xi}, Y\right)=E\left(T_{\xi}, Y\right)+\gamma \sum_{m=1}^{M} \sum_{n=1}^{N}\left(\left(\xi_{m n}^{x}\right)^{2}+\left(\xi_{m n}^{y}\right)^{2}\right),
$$

where $\gamma$ provides a relative weighting between the two penalty terms.

The output of the deformation process is a single objective function value in the range $[0,1]$, with zero indicating a perfect match with no deformation. It is important to note that this objective value is not symmetric, that is, the objective value from matching a template derived from image $i$ to image $j$ will not necessarily be the same as that of matching template $j$ to image $i$.

The above process deforms the template so that it corresponds as closely as possible to edges in the target images. In practice, however, this is not sufficient for matching, as templates of topologically simple characters such as " 1 " and " 0 " can often be mapped on to the edges of any target image. Because of this, we also calculate binary matching coefficients between the target image and the interior of the deformed outline. The Jaccard measure (selected on the basis of its good performance in the evaluation of Tubbs [15]) is used to gauge the similarity between two binary digit images. The Jaccard measure $J_{i j}$ between two binary images $i$ and $j$ is defined as 


$$
J_{i j}=\frac{b_{01}+b_{10}}{b_{00}+b_{01}+b_{10}},
$$

where $b_{00}$ is the number of points which are object pixels in both images, and $b_{10}$ and $b_{01}$ count the pixels which are background in one image and object in the other. Note that this measure is actually the standard Jaccard measure [5] subtracted from one, so that lower values indicate better matches, just as in the objective function defined in (7).

The dissimilarity between two binary images (a template $i$ and a target image $j$ ) is now computed as a weighted sum of the two dissimilarities defined in (7) and (8).

$$
D_{i j}=\alpha \mathrm{O}_{i j}+(1-\alpha) J_{i j}, \quad 0 \leq \alpha \leq 1 .
$$

(Note that $O(T \xi, Y)$ in (7) is here denoted as $O_{i j}$.) The weight $\alpha$ needs to be specified by the user. With this measure, a smaller value of $D_{i j}$ indicates more similar images. Fig. 3 shows the results of two deformations, one with images from the same class and one with images of differing classes. Table 1 gives the value of $D_{i j}$ for these two pairs, with various weight values.

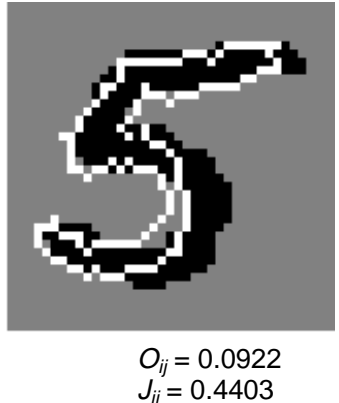

(a)

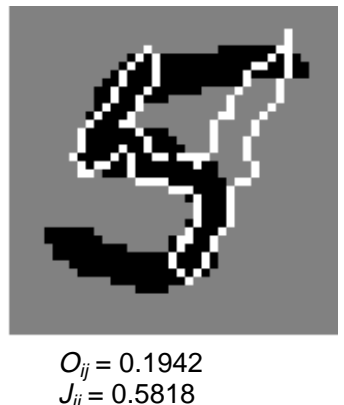

(b)
Fig. 3. Deformed template superimposed on target image, with dissimilarity measures. (a) Template from the same class as target. (b) Template from a different class.

TABLE 1

DISSIMILARITY VALUES FOR THE IMAGE PAIRS OF FIG. 3, FOR VARIOUS VALUES OF WEIGHT $\alpha$

\begin{tabular}{|c|cc|}
\hline$\alpha$ & $D_{i j}$ pair $(\mathrm{a})$ & $D_{i j}$ pair $(\mathrm{b})$ \\
\hline 1 & 0.0922 & 0.1942 \\
$1 / 2$ & 0.2662 & 0.3880 \\
0 & 0.4403 & 0.5818 \\
\hline
\end{tabular}

\section{Multidimensional Scaling for Feature EXTRACTION}

At this point we have defined two dissimilarity measures $O_{i j}$ and $J_{i j}$ between a pair of character images, and can calculate an $n \times n$ proximity matrix for a set of $n$ input images. To apply many standard pattern classification techniques, however, we need an $n \times d$ pattern matrix-a set of $d$ features for each of the $n$ patterns.

Multidimensional scaling [7] is a well-known technique to obtain an appropriate representation of the patterns from the given proximity matrix. Given an $n \times n$ input matrix of interpattern distances, multidimensional scaling creates an $n \times d$ pattern matrix; embedding the $n$ patterns as points in a $d$-dimensional space, trying to keep the distances between patterns as close to the input dissimilarity matrix as possible. For a given $d$, the algorithm minimizes a stress value, which measures the similarity between the given proximity matrix and the interpoint distances of the output pattern matrix. The pattern matrices produced by two sample multidimensional scaling runs (corresponding to the starred entries of Table 2) are shown in Figs. 4 and 5.

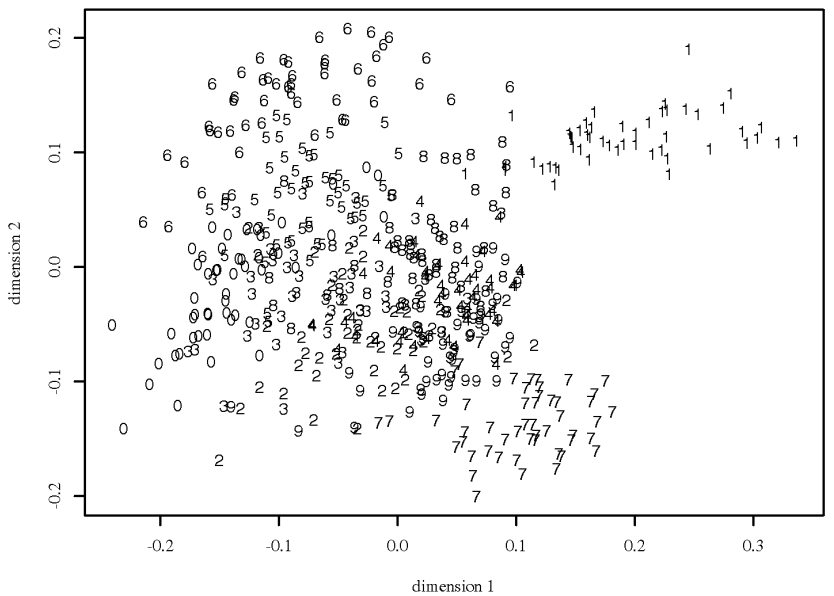

Fig. 4. Two-dimensional pattern matrix produced by multidimensional scaling, with $\alpha=1 / 2$.

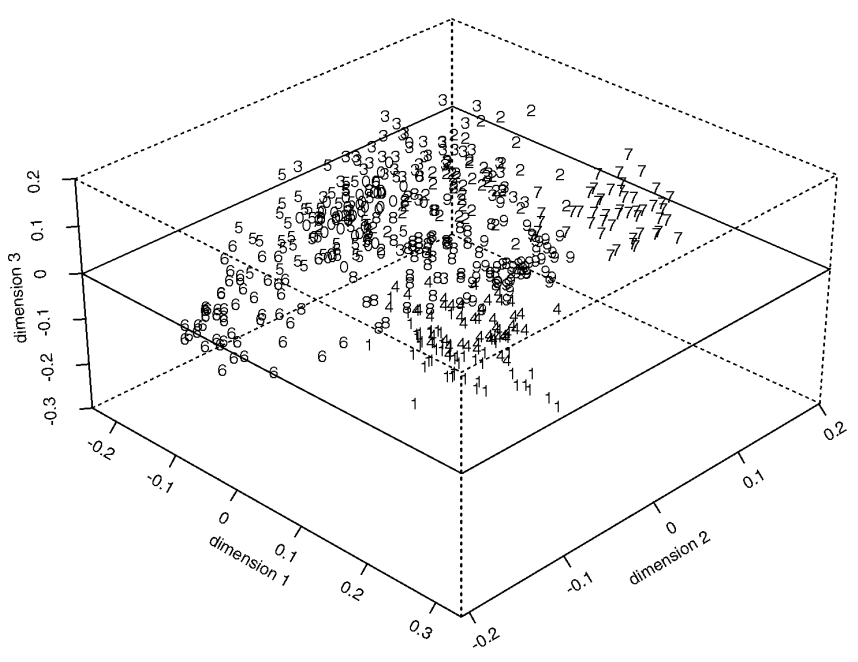

Fig. 5. Three-dimensional pattern matrix produced by multidimensional scaling, with $\alpha=1 / 2$.

It is expected that given a meaningful set of interpattern distances as input, the multidimensional scaling algorithm [12] will generate a pattern matrix that represents pattern classes as compact and isolated clusters in a feature space. We have applied multidimensional scaling to the dissimilarity matrices produced by the deformable template method, and used a nearest-neighbor (NN) classifier to evaluate the quality of the resulting pattern matrix or the representation space.

The stress values obtained using this procedure for different values of $d$ (dimensionality of the representation space) are given in Table 2 and plotted in Fig. 6. Three different values of $\alpha$ were used: 0,1 , and $1 / 2$. These correspond to using the objective function value $O_{i j}$ only, the Jaccard measure $J_{i j}$ only, and an equally weighted sum of the two. Each dissimilarity matrix was averaged with its transpose to produce a symmetric distance matrix. Due to computational limitations, only 500 of the 2,000 patterns in the database were used in this analysis. So, an attempt was made to embed 500 patterns in feature spaces of dimensionality ranging from two to nine. Stress generally decreases as $d$ increases over this range. It is generally suggested that a stress value below 0.05 corresponds to a "good" representation. The quality of the derived representation will be determined based on the classification results in the next section. 
TABLE 2

MULTIDIMENSIONAL SCALING STRESS VALUES, FOR VARIOUS DISSIMILARITY MEASURES AND DIMENSIONALITIES

\begin{tabular}{|c|cccccccc|}
\hline & \multicolumn{7}{|c|}{ \# of dimensions, $d$} \\
\cline { 2 - 9 }$\alpha$ & 2 & 3 & 4 & 5 & 6 & 7 & 8 & 9 \\
\hline 1 & 0.2509 & 0.1501 & 0.11588 & 0.08897 & 0.07742 & 0.07300 & 0.07179 & 0.07561 \\
$1 / 2$ & $0.3614^{*}$ & $0.2500^{*}$ & 0.18922 & 0.15244 & 0.12255 & 0.09837 & 0.08375 & 0.07016 \\
0 & 0.3976 & 0.2968 & 0.22817 & 0.18680 & 0.15400 & 0.13094 & 0.11287 & 0.09934 \\
\hline
\end{tabular}

Pattern matrices for the entries marked with * are plotted in Figs. 4 and 5.

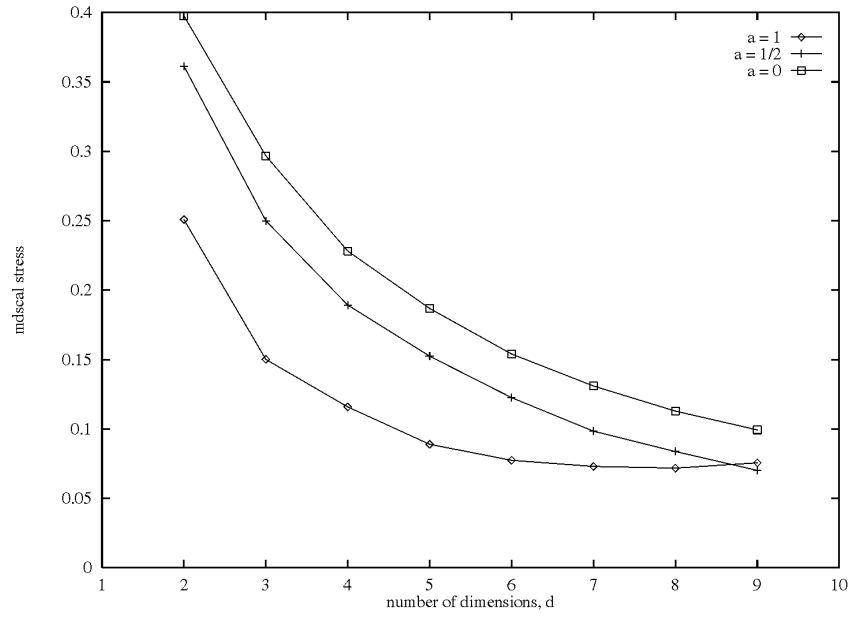

Fig. 6. Plot of multidimensional scaling stress vs. number of features.

\section{Classification Results}

The results presented here are based on a 2,000 digit sample from NIST Special Database 1 and an independent 2,000 digit sample from IBM Almaden Research Center. First, we describe the classification methodology. Each character in the NIST database is a $32 \times 32$ binary image. A four-pixel-wide border was placed around each image to allow the deformation process some room to adjust the template in, so the actual image size used was $40 \times 40$ pixels. We use the dissimilarity value $D_{i j}$ in (9) to classify each target image. A leave-one-out approach is used, with two different ways of calculating the dissimilarity value. In the first ("asymmetric"), the unknown image is classified by taking it as the target image, and each of the other 1,999 images as templates in turn. The unknown image is assigned to the class of the template with the minimum dissimilarity value. The second ("symmetric") method also compares the unknown image with the other 1,999 images but instead of treating the unknown image as the target and the known image as the template, it performs the deformation both ways and averages the results. While the second method gives better results, it has the disadvantage of requiring twice as many deformations to classify an unknown image.

Table 3 gives the classification accuracies for both the NIST data and the IBM data for different values of the weight $\alpha$. The asymmetric method is especially poor when only the objective function value is used. Digits with simple shapes ( 1 and 0 ) can be deformed so their edges fit quite well with some of the edges of the target image, but without the entire target image being covered. This produces a low objective function value which leads to a misclassification of digits as 1 s or 0 s. Forcing each digit's outline to fit the other's (symmetric method) and/or measuring the overlap after deformations (Jaccard coefficient) corrects this tendency. The 15 NIST images misclassified using the symmetric dissimilarity with $\alpha=1 / 2$ are shown in Fig. 7. Some of these images are very difficult to classify, even by a human expert.
TABLE 3

Classification AcCuRACIES USING the DissimilaRIty VALUe $D_{i j}$

\begin{tabular}{|c|cc|cc|}
\hline \multirow{2}{*}{$\alpha$} & \multicolumn{2}{|c|}{ NIST data } & \multicolumn{2}{c|}{ IBM data } \\
& asymmetric & symmetric & asymmetric & symmetric \\
\hline 1 & 952 & 1,873 & 1,256 & 1,661 \\
& $(47.60 \%)$ & $(93.65 \%)$ & $(62.80 \%)$ & $(83.05 \%)$ \\
$1 / 2$ & 1,957 & 1,985 & 1,802 & 1,845 \\
& $(97.85 \%)$ & $(99.25 \%)$ & $(90.10 \%)$ & $(92.25 \%)$ \\
0 & 1,951 & 1,971 & 1,787 & 1,873 \\
& $(97.55 \%)$ & $(98.55 \%)$ & $(89.35 \%)$ & $(93.65 \%)$ \\
\hline
\end{tabular}

Each dataset contains 2,000 character images.

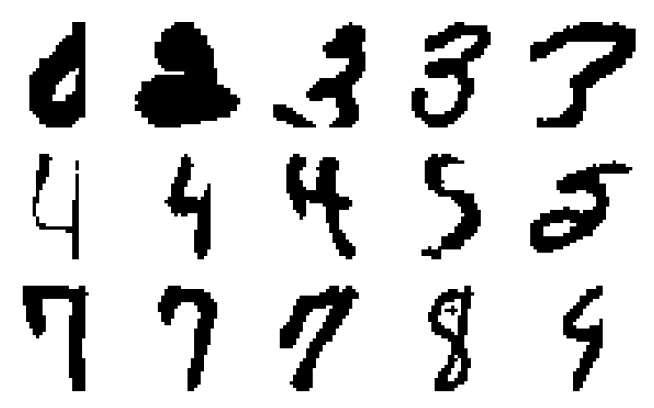

(a)

$\begin{array}{lllll}6 & 6 & 2 & 8 & 7 \\ 6 & 9 & 7 & 9 & 2 \\ 9 & 9 & 9 & 9 & 4\end{array}$

(b)

Fig. 7. Misclassified digits by the best classifier of Table 3. (a) The 15 input images that were misclassified. (b) The classes assigned by the classifier.

Classification was also done by using a nearest-neighbor algorithm on the pattern matrix produced by the multidimensional scalings in Section 4. A leave-one-out approach was used. These results are given in Table 4 and plotted in Fig. 8. The best 1NN (one nearest neighbor) recognition rate obtained was 97.0 percent, using $\alpha=1 / 2$, with nine dimensions. While this technique is impractical for use as a classifier in a production system (the computationally expensive multidimensional scaling algorithm would have to be applied for each digit to be classified), it does illustrate the existence of a relatively small set of features that give good classification performance with a simple classifier such as $1 \mathrm{NN}$. These results should motivate us to search for a good representation space for handwritten digits.

The computational requirements of our deformable template approach to digit classification are high. To classify a single character against the database of 2,000 images, using the asymmetric dissimilarity would require running the deformation process 2,000 times, which takes approximately 38 CPU minutes on a Sun Ultra 1 . Use of the symmetric dissimilarity doubles the necessary computational effort. To use the NN method as a classifier would require additionally rerunning the multidimensional scaling 


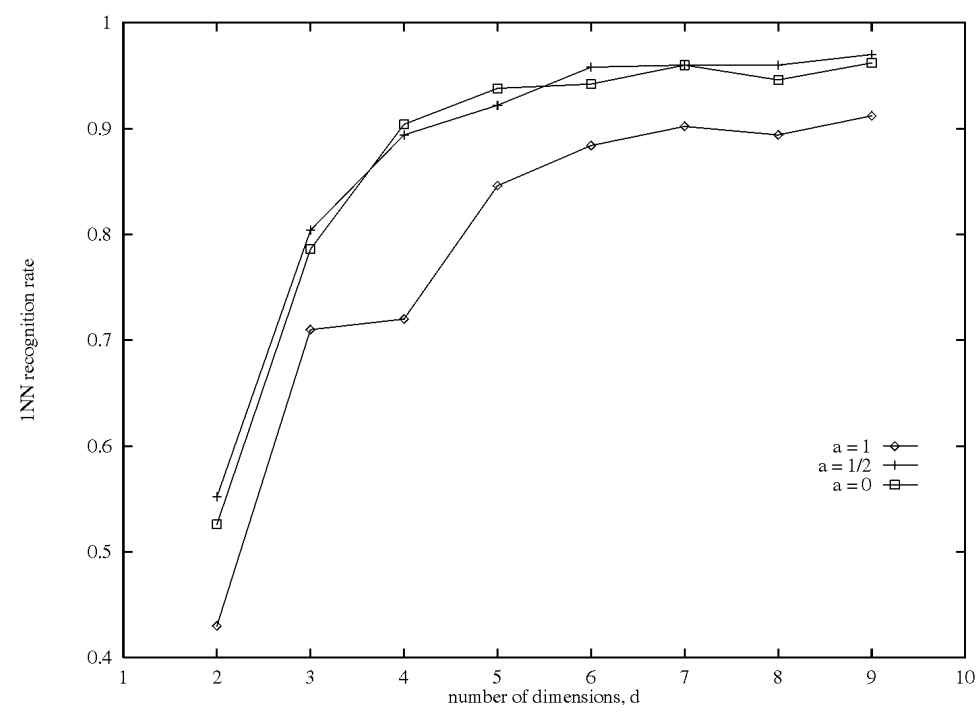

Fig. 8. Plot of $1 \mathrm{NN}$ recognition accuracy vs. number of dimensions.

TABLE 4

Results of 1NN Classifier Applied to the PATtern MATRIX DERIVEd From Multidimensional Scaling

\begin{tabular}{|c|cccccccc|}
\hline$\alpha$ & 2 & 3 & 4 & 5 & 6 & 7 & 8 & 9 \\
\hline 1 & 0.430 & 0.710 & 0.720 & 0.846 & 0.884 & 0.902 & 0.894 & 0.912 \\
$1 / 2$ & 0.552 & 0.804 & 0.894 & 0.922 & 0.958 & 0.960 & 0.960 & 0.970 \\
0 & 0.526 & 0.786 & 0.904 & 0.938 & 0.942 & 0.960 & 0.946 & 0.962 \\
\hline
\end{tabular}

process for the 2,000 database images plus the test images. Obviously, this does not make for a practical classifier.

One way to reduce this computational burden would be to reduce the size of the training set, by selecting a small number of images to serve as prototypes for the whole class. One approach would be to cluster the patterns of each class, and select a representative of each cluster. To implement this strategy, we performed a complete-link hierarchical clustering [5] on the patterns of each class, independently. The resulting dendrogram was cut to form $p$ clusters. To choose a representative from each resulting cluster, the sum of dissimilarities from each member to all other members of the cluster was computed. The member with the minimum such sum is chosen. In this way, $p$ prototype images from each class are chosen, $10 p$ images in all for the digit database.

The prototype set is tested using the minimum dissimilarity classification method, as in Table 3 . The symmetric dissimilarity value is used. Instead of a leave-one-out method as above, a holdout method is used-the prototypes forming the training set are selected from the NIST data set, and the IBM data is used as the test set. The classification accuracies using this method, for different values of $p$, are shown in Table 5. It is reasonably robust even considering the marked differences between the NIST and IBM data sets (see Fig. 1), most notably in image size $(32 \times 32$ for NIST, $16 \times 24$ for IBM).

TABLE 5

CLASSIFICATION ACCURACIES OF LEAST DISSIMILAR PROTOTYPE PATTERN MATCHING

\begin{tabular}{|c|c|c|c|c|c|}
\hline \multirow[b]{2}{*}{$\alpha$} & \multicolumn{4}{|c|}{ \# of prototypes per class, $p$} & \multirow{2}{*}{$\begin{array}{c}\text { full data- } \\
\text { base } \\
(p=200)\end{array}$} \\
\hline & 5 & 10 & 20 & 30 & \\
\hline 1 & 0.369 & 0.359 & 0.423 & 0.456 & 0.479 \\
\hline $1 / 2$ & 0.736 & 0.774 & 0.849 & 0.843 & 0.925 \\
\hline 0 & 0.694 & 0.760 & 0.840 & 0.847 & 0.928 \\
\hline
\end{tabular}

\section{SUMmarY}

We have used a deformable template approach for the purpose of handprinted digit recognition. The deformation system used represents one binary image in terms of its contour, and then iteratively computes parameters of a continuous displacement function in order to map the contour template as closely as possible onto the edges of the other binary target image.

Two dissimilarity measures between character image pairs have been defined: a measure of the amount of deformation needed, and the Jaccard binary matching coefficient between the target image and the deformed template image. Classifying each image using the minimum dissimilarity to all the other templates produced over 99 percent accuracy on a 2,000 image data derived from the NIST database. Additional experiments were also done on an independent dataset available from IBM. Results of multidimensional scaling demonstrate that it is possible to obtain a good low-dimensional representation space for hand printed digits based on the deformation process.

Future work will focus on reducing the computational requirements of this method, through faster deformation algorithms and better selection of representative prototypes from the training set. We are also studying how to learn the deformations which are more appropriate for individual digits.

\section{REFERENCES}

[1] R.G. Casey, "Moment Normalization of Handprinted Characters," IBM J. Research and Development, pp. 548-557, Nov. 1970.

[2] K.-W. Cheung, D.-Y. Yeung, and R.T. Chin, "A Unified Framework for Handwritten Character Recognition Using Deformable Models," Proc. Second Asian Conf. Computer Vision, vol. 1, pp. 344348, 1995.

[3] P. Gader, B. Forester, M. Ganzberger, A. Gillies, B. Mitchell, M. Whalen, and T. Yocum, "Recognition of Handwritten Digits Using Template and Model Matching," Pattern Recognition, vol. 24, no. 5 , pp. $421-431,1991$. 
[4] J. Geist et al., The Second Census Optical Character Recognition Systems Conf., Nat'l Inst. of Standards and Technology, NISTIR 5452, May 1988.

[5] A.K. Jain and R.C. Dubes, Algorithms for Clustering Data. Englewood Cliffs, N.J.: Prentice Hall, 1988.

[6] A.K. Jain, Y. Zhong, and S. Lakshmanan, “Object Matching Using Deformable Templates," IEEE Trans. Pattern Analysis and Machine Intelligence, vol. 18, no. 3, pp. 267-278, Mar. 1996.

[7] J.B. Kruskal, "Multidimensional Scaling and Other Methods for Discovering Structure," K. Enslein, A. Ralson, and H.S. Wilf, eds., Statistical Methods for Digital Computers, pp. 296-339. New York: John Wiley \& Sons, 1997.

[8] L. Lam and C.Y. Suen, "Structural Classification and Relaxation Matching of Totally Unconstrained Handwritten Zip-Code Numbers," Pattern Recognition, vol. 21, no. 1, pp. 19-31, 1988.

[9] H. Nishida, "A Structural Model of Shape Deformation," Pattern Recognition, vol. 28, no. 10, pp. 1,611-1,620, 1995.

[10] M. Revow, C.K.I. Williams, and G.E. Hinton, "Using Generative Models for Handwritten Digit Recognition," IEEE Trans. Pattern Analysis and Machine Intelligence, vol. 18, no. 6, pp. 592-606, June 1996.

[11] P.Y. Simard, Y. Le Cun, and J.S. Denker, “Memory-Based Character Recognition Using a Transformation Invariant Metric," Proc. 12th Int'l Conf. Pattern Recognition, pp. 262-267, Oct. 1994.

[12] Statistical Sciences, Inc., S-PLUS 3.2, 1993.

[13] C.Y. Suen, R. Legault, C. Nadal, M. Cheriet, and L. Lam, "Building a New Generation of Handwriting Recognition Systems," Pattern Recognition Letters, vol. 14, pp. 303-315, Apr. 1993.

[14] O.D. Trier, A.K. Jain, and T. Taxt, "Feature Extraction Methods for Character Recognition-A Survey," Pattern Recognition, vol. 29, no. 4, pp. 641-662, 1996.

[15] J.D. Tubbs, "A Note on Binary Template Matching," Pattern Recognition, vol. 22, no. 4, pp. 359-365, 1989.

[16] T. Wakahara, "Shape Matching Using LAT and Its Application to Handwritten Numeral Recognition," IEEE Trans. Pattern Analysis and Machine Intelligence, vol. 16, no. 6, pp. 618-629, June 1994. 